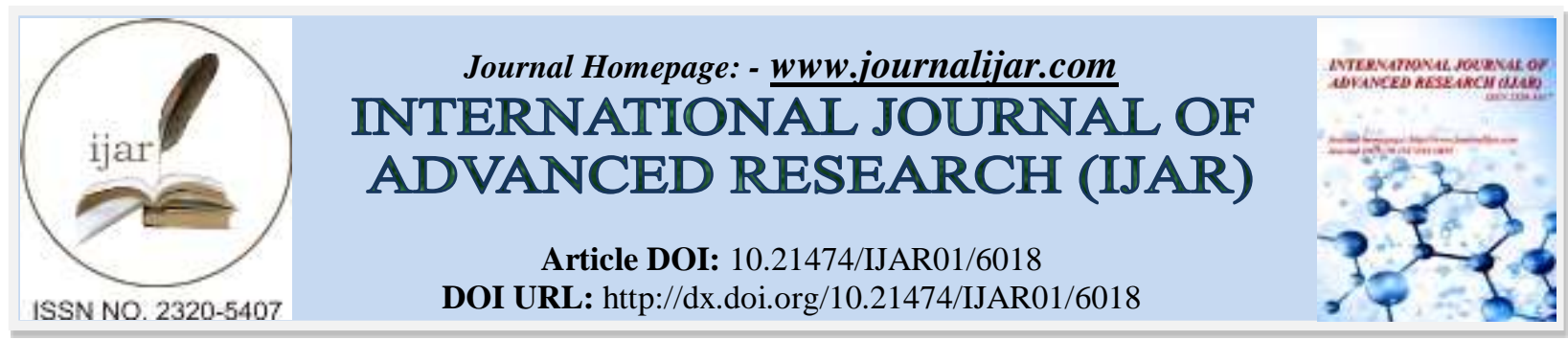

RESEARCH ARTICLE

\title{
TRANSVERSUS ABDOMINIS PLANE (TAP) BLOCK VERSUS LOCAL ANESTHETIC WOUND INFILTRATION (LWI) FOR POST TOTAL ABDOMINAL HYSTERECTOMY (TAH) PAIN RELIEF.
}

Mohamed Farag El Sherbeny ${ }^{1}$ and Elsayed Mohamed Abdelzaam ${ }^{2}$.

1. Assistant Professor of Obstetrics and Gynecology, Benha Faculty of Medicine, Benha University, Egypt.

2. Lecturer of Anesthesia \& I.C.U, Benha University Hospital, Benha University Egypt.

\section{Manuscript Info}

(.........................

Manuscript History

Received: 10 October 2017

Final Accepted: 12 November 2017

Published: December 2017

Key words:-

Total abdominal hysterectomy, visual analogue scale, Bupivacaine, transversus abdominis plane block, local anesthetic wound infiltration, opioids consumption.

\section{Abstract}

Aim: to compare bilateral ultrasound guided transversus abdominis plane (TAP) block with single - shot - local anesthetic subcutaneous and sub facial wound infiltration (LWI) for analgesia after total abdominal hysterectomy (TAH) under general anesthesia.

Patients and methods: This prospective randomized, parallel group, concealed allocation, patients and observers blinded superiority trial, conducted at Benha University Hospital, including 87 women undergoing TAH, 43 women received $40 \mathrm{ml} 0.25$ bupivacaine as bilateral ultrasound guided TAP block and 44 women received $40 \mathrm{ml}$, $0.25 \%$ bupivacaine as subcutaneous and sub facial local wound infiltration at the end of TAH performed under general anesthesia. Main outcomes were total cumulative Nalbuphine consumption and post TAH pain at rest and with movement using visual analogue scale (VAS) as well as incidence of nausea, vomiting, other analgesic requirements, time spent in post anesthesia care unit (PACU), time to get out of bed, deepest sedation, patient satisfaction regards analgesia in First 24 hour postoperative.

Results: Total Nalbuphine consumption were significantly lower in TAP block group at 2, 4, 8, 12, 24 hours and at discharge $(\mathrm{P}<0.0001)$. The mean post TAH pain VAS score both at rest and on movement were significantly lower in TAP block group at PACU, 2h, 8h, 12h (P < $0.05)$ as well as at $4 \mathrm{~h}$ on movement $(\mathrm{P}<0.05)$. Also, total non-steroidal anti-inflammatory drugs (NSAIDs) consumption were lower in TAP block up to twenty-four hours postoperatively $(\mathrm{P}<0.005)$, time to get out of bed $(P=0.03)$ and time to first flatus $(P=0.0002)$.

Conclusion: Bilateral ultrasound guided TAP block is superior to local anesthetic wound infiltration in analgesia for post TAH pain as Tap block was associated with reduced both rest and on movement postoperative pain and total cumulative opioids analgesia as well as promotes early mobilization and gut recovery.

Copy Right, IJAR, 2017,. All rights reserved. 


\section{Introduction:-}

Hysterectomy is the most common major gynecological operation ${ }^{(1)}$. The post total abdominal hysterectomy (TAH) pain and discomfort is more obvious and sever when compared to other routes for hysterectomy, despite that, most worldwide hysterectomies were performed abdominally ${ }^{(1)}$. To control the post TAH pain, women usually utilized excessive amount of opioid analgesics, although it is associated with several complications as nausea, vomiting, sedation, itching, inhibition of intestinal mobility and respiratory depression ${ }^{(2,3)}$ as well as postoperative cognitive dysfunction in elder women and resulting delayed mobilization and rehabilitation ${ }^{(4)}$. Thus, alternative approaches, which reduce the requirement for strong opioids postoperatively, are required.

Abdominal hysterectomy is associated with two types of pain, a visceral origin dull nauseating continuous pain, with well response to opiates and a somatic origin sharper pain induced by coughing and movement, with poor response to opioids ${ }^{(5,6)}$. Thus, multimodal analgesia after TAH is highly needed ${ }^{(5,6)}$.

Several strategies to control somatic component of post TAH pain have been trialed including transversus abdominis plane (TAP) block and local anesthetic wound infiltration (LWI) ${ }^{(7,8,9,10,11)}$.

Transversus abdominis plane (TAP) block is a regional analgesic technique, affecting the nerves supplying the anterior abdominal wall, found to be effective after abdominal and pelvic surgeries ${ }^{(7)}$. Single shot or continuous infiltration of local anesthetic into surgical wound has been trailed with varying degree of efficacy in postoperative analgesia after $\mathrm{TAH}^{(8,9,10,11)}$.

Both TAP block $^{(7)}$ and LWI ${ }^{(11)}$ shown to be superior to placebo for postoperative analgesia after TAH, however, it is unknown which of them provides better postoperative analgesia after TAH because of lack of randomized clinical trials compared TAP block to LWI in post TAH pain. Few studies comparing TAP block and LWI for postoperative analgesia after cesarean section were existed ${ }^{(12,13,14,15)}$.

The objective of this trial was to compare bilateral ultrasound guided TAP block with single shot local anesthetic wound infiltration (LWI) for analgesia after TAH performed under general anesthesia. We hypothesized that TAP block would reduce postoperative total cumulative Naluphine consumption.

\section{Patients and Methods:-}

This prospective, randomized, parallel group, concealed allocation, patients and observers blinded superiority trial was conducted at obstetrics and gynecology department of Benha University Hospital, Al Kalubia, Egypt from October 2015 to December 2016. The study protocol was approved by Benha Faculty of Medicine ethics committee and informed written consents were taken from participants in this trial. All women scheduled for TAH for benign indications between October 2015 and December 2016 were asked to participate. Exclusion criteria were allergy to either amide local anesthetics or opiates, ongoing treatment for depression, weight under 50kg (Dose toxicity), daily consumption of pain killers, fibromyalgia, dementia or mental retardation to a degree which would interfere with data collection.

Women were recruited sequentially and assigned to transversus abdominis plane (TAP) block or local anesthetic wound infiltration (LWI) at random in 1:1 ratio. The trial statistician created the randomized treatment allocation schedule by using a computer random number generator. The treatment allocation schedule was stored by the gynecologist (M.F.E) and the point of randomization occurred when the women was asked to enter the operation room. The study subjects and the outcomes assessors were blinded to group allocation.

Under general anesthesia (including Propofol or thiopental as induction agent, cistracurium as muscle relaxant, isoflurane as inhalational anesthetic, nalbuphine as an analgesic and neostigmine as muscle relaxant reverse, women were positioned and TAH was performed in usual way through transverse pfannenstiel incision, before wound closure in LWI group, $40 \mathrm{ml}$ of bupivacaine $0.25 \%$ were injected by gynecologist both in upper and lower wound edges, both subcutaneous and sub facial. While after wound closure in TAP block group, a trained anesthesiologist (E.M.A) with ultrasound guided TAP block inject $20 \mathrm{ml}$ of $0.25 \%$ bupivacaine on either side with aids of $4-12$ MHZ linear array transducer (Mindray 2200 plus, China) placed transversely between iliac crest and costal margin in anterior axillary line and slid medial to lateral to visualize the external oblique, internal oblique and transversus abdominis muscles. A 22 - gauge spinal needle was introduced from medial to lateral in plane with the ultrasound 
probe and local anesthetic was injected under direct visualization in the plane between the transversus abdominis muscle and the fascia deep to internal oblique muscle. Women were monitored for degree of sedation, respiratory and hemodynamic stability, nausea, vomiting, pain at post anesthesia care unit (PACU). When women were finally awake and vitally stable; they were transferred to post-operative word. Nalbuphine HCL (Nalufin ${ }^{\circledR} 20 \mathrm{mg} / \mathrm{ml}$, AMON pharmacoceutical Co. SAE, El Obour City, Cairo, Egypt) $20 \mathrm{mg}$ was diluted in $20 \mathrm{ml}$ saline and $5 \mathrm{ml}(5 \mathrm{mg}$ ) was given on patient request if visual analogue scale (VAS) of pain $\geq 40 \mathrm{~mm}$ at rest at postoperative word while tenoxicam (Epicotil ${ }^{\circledR} 20 \mathrm{mg}$ vial E.I.P.I.Co. $10^{\text {th }}$ of Romadan City - Egypt) $20 \mathrm{mg}$ intravenously was given if patient expressing pain on walking $\geq 40 \mathrm{~mm}$ on VAS at postoperative ward.

The primary outcomes were postoperative total cumulative Nalbuphine consumption and postoperative pain severity both at rest and on movement (coughing and hip flexion) evaluated by VAS score ranging from $0-100 \mathrm{~mm}$, where 0 indicates no pain and $100 \mathrm{~mm}$ indicates the worst pain at PACU, 2, 6, 12, 24 hour postoperatively, while the secondary outcomes were level of sedation assessed using a $4-$ point scale $(0=$ awake and alert, $1=$ minimally sedated, responds to speech, $2=$ moderately sedated, responds to tactile stimulation and $3=$ deeply sedated, arousable only by painful stimulation, the deepest level of sedation reached by each women during the 24 hour period postoperatively was recorded, presence of Nalbuphine - related side effects and tenexicam related side effects, presence of nausea, vomiting, time to get out of bed, hospital stay and patient satisfaction from postoperative analgesia assessed at 24 hour postoperatively using a 5 - point scale $(5=$ very satisfied, $4=$ satisfied, $3=$ fair, $2=$ unsatisfied and $1=$ very unsatisfied).

Before starting this trial, we performed a pilot study to assess the average of total postoperative cumulative Nalbuphine consumption after TAH with subcutaneous and sub facial local Bupivacine $0.25 \%, 40 \mathrm{ml}$ wound infiltration and was found to be $48.5 \pm 22.6$. Assuming $5 \%$ level of significance (type I or alpha error $=0.05$ ) and $80 \%$ power (type II or Beta error $=0.2$ ) and using the 2 - tailed student $t$ test, 38 women were required in each group to detect a $14.55 \mathrm{mg}(30 \%)$ reduction in mean total postoperative cumulative Naluphine consumption, which was considered the minimal clinically significant effect by TAP block over LWI. To compensate for $20 \%$ dropout, a total of 92 women needed for this study.

Statistical analysis was by modified intention to treat analysis, where only women who randomized and who received all of the study intervention were included in the final analysis and were performed by free trial MedCalc easy - to - use stastical software for window desktop (www.Medcalc.org) 2017 MedCalc, software, bvba ${ }^{(16)}$. Continuous variable is presented in terms of means, standard deviations and ranges while categorical variable is presented in terms of frequencies and percents as appropriate. Fisher's exact test was used to compare categorical variables as incidence of nausea, vomiting, pruritis. Independent sample student's $t$ test was used to compare continuous variables as baseline demographic and clinical criteria as amount of analgesia, operative time, visual analogue scale score and blood loss. P values and mean difference with 95\% confidence interval (CIs) were used to determine significance, $\mathrm{P}<0.05$ was considered statistically significant.

\section{Results:-}

In the present study, one hundred fifteen women were evaluated for eligibility, ninety-two women were eligible and randomized to either ultrasound guided bilateral TAP block or local anesthetic wound infiltration, forty-six in each group. Five women didn't receive allocated intervention as randomized, three in TAP block group and two in LWI group due to necessity to do additional surgery other than simple TAH. Forty-three women received TAP block and forty-four women received LWI as randomized, all these eighty-seven women were included in the primary analysis (Figure I).

Table (1) presents participants demographic and clinic - surgical criteria and shows there were no statistically significant difference between both groups.

Table (2) and table (3) present pain difference between TAP block and LWI at rest and on movement respectively, and shows that pain with movement statistically significantly lower in TAP block than LWI at PACU, $2 \mathrm{~h}, 4 \mathrm{~h}, 8 \mathrm{~h}$, $12 \mathrm{~h}$ postoperatively while pain at rest were significantly lower at PACU, $2 \mathrm{~h}, 8 \mathrm{~h}$ only. 
Table (4) shows that cumulative Nalbuphine consumption $(\mathrm{mg})$ were significantly lower at $2 \mathrm{~h}, 4 \mathrm{~h}, 8 \mathrm{~h}, 24 \mathrm{~h}$ and at discharge in TAP block group than LWI group. Also, cumulative parenteral NSAIDs consumption were significantly lower in TAP block group than LWI group at $2 \mathrm{~h}, 4 \mathrm{~h}, 8 \mathrm{~h}, 12 \mathrm{~h}, 24 \mathrm{~h}$, but the total consumption was not.

Table (5) shows that time to get out of bed, time to first flatus were significantly lower in TAP block group while there was no significance difference as regards PACU time, hospital stay, nausea, vomiting, pruritis, patient satisfaction, deepest level of sedation.

No apparent complications were recorded in both groups as a result of $40 \mathrm{ml}$ bupivacaine $0.25 \%$ injection either as TAP block or LWI. Also, there was no complication from TAP block procedures.

Fig (I):- Participants flow in TAP block versus LWI for post TAH pain trial

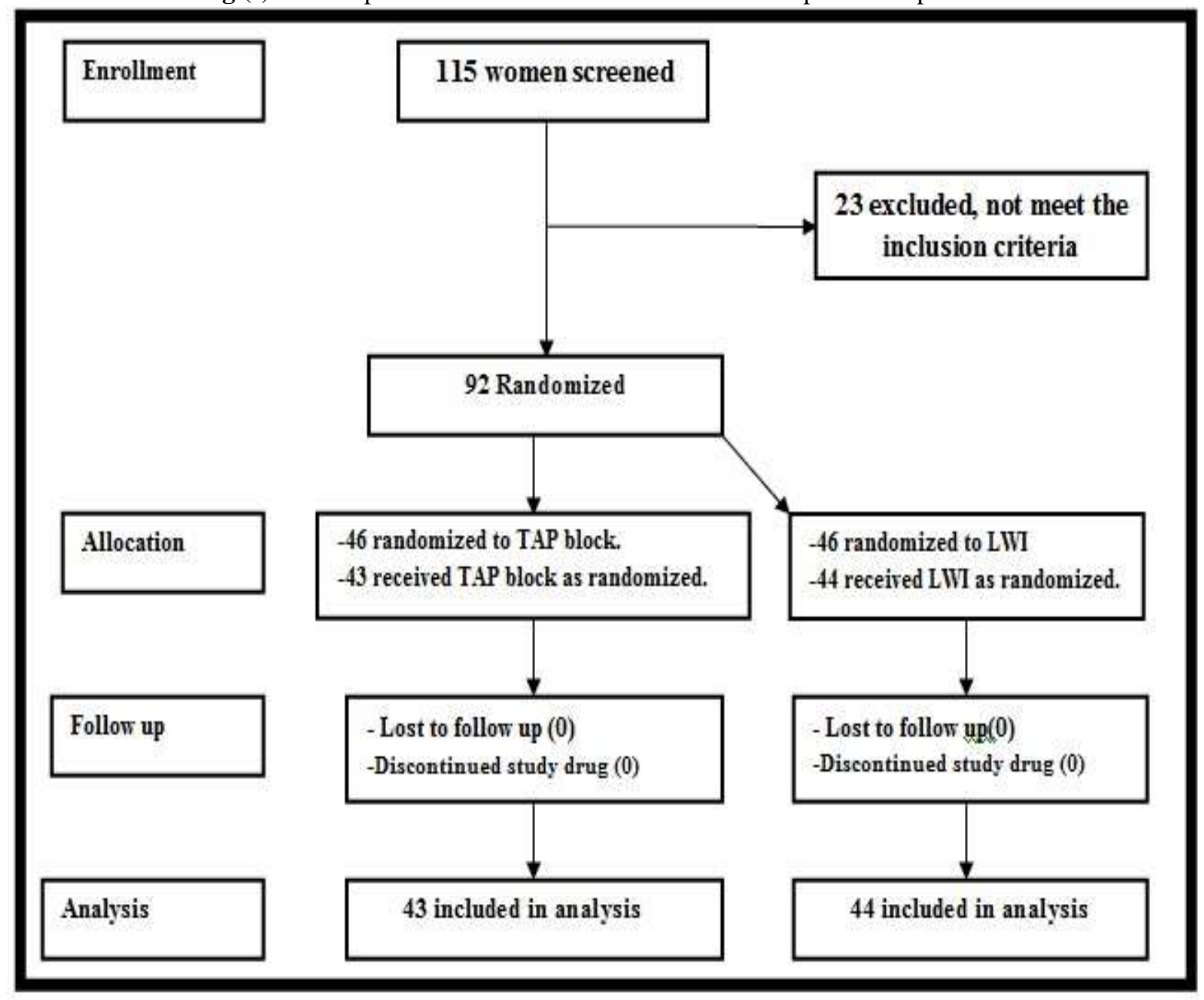

Abbreviation:-

TAP: transversus abdominis plane, LWI: local anesthetic wound infiltration, TAH: Total abdominal hysterectomy. 
Table (1):- Demographic and clinic - surgical criteria of women receiving 40ml $0.25 \%$ bupivacaine as TAP block or LWI for post TAH pain.

\begin{tabular}{|c|c|c|c|c|c|}
\hline Variable & $\begin{array}{c}\text { TAP } \\
(n=43)\end{array}$ & $\begin{array}{c}\text { LWI } \\
(n=44)\end{array}$ & $\begin{array}{c}\Delta \\
\text { mean }\end{array}$ & $95 \%$ C. I & $P$ value \\
\hline - Age (year)* & $\begin{array}{c}47.9 \pm 4.6 \\
(42-63)\end{array}$ & $\begin{array}{c}48.4 \pm 3.9 \\
(43-61)\end{array}$ & 0.5 & $(-1.3,2.3)$ & $=0.5$ \\
\hline - BMI $\left(\mathrm{kg} / \mathrm{m}^{2}\right) *$ & $\begin{array}{c}31.3 \pm 3.9 \\
(24.6-38.9)\end{array}$ & $\begin{array}{c}30.8 \pm 5.1 \\
(24.2-37.8)\end{array}$ & -0.5 & $(-2.4,1.4)$ & $=0.6$ \\
\hline \multicolumn{6}{|l|}{ - Indications of TAH ${ }^{* * *}$} \\
\hline - PMB & $22(51.2 \%)$ & $24(54.5 \%)$ & $3.3 \%$ & $(-18.7,25.0)$ & $=0.7$ \\
\hline -Precancerous conditions & $6(13.9 \%)$ & $5(11.3 \%)$ & $2.6 \%$ & $(-13.1,18.4)$ & $=0.7$ \\
\hline - Fibroids & $10(23.2 \%)$ & $9(20.4 \%)$ & $2.8 \%$ & $(-15.9,21.4)$ & $=0.7$ \\
\hline - Adenomyosis & $5(11.6 \%)$ & $6(13.5 \%)$ & $2 \%$ & $(-13.8,17.7)$ & $=0.7$ \\
\hline \multicolumn{6}{|l|}{-Concomitant comorbidity } \\
\hline - Diabetes & $8(18.6 \%)$ & $9(20.4 \%)$ & $1.8 \%$ & $(-16.4,19.8)$ & $=0.8$ \\
\hline - Hypertension & $6(13.9 \%)$ & $8(18.1 \%)$ & $4.2 \%$ & $(-12.9,21.09)$ & $=0.5$ \\
\hline - Others & $2(4.6 \%)$ & $3(6.8 \%)$ & $2.2 \%$ & $(-10.1,14.7)$ & $=0.6$ \\
\hline - Uterine weight $(\mathrm{gm})^{*}$ & $\begin{array}{c}185 \pm 60 \\
(80-360)\end{array}$ & $\begin{array}{c}195 \pm 50 \\
(90-390)\end{array}$ & 10 & $(-13.5,33.5)$ & $=0.4$ \\
\hline - Operative time $(\mathrm{min})^{*}$ & $\begin{array}{c}110 \pm 40 \\
(75-145)\end{array}$ & $\begin{array}{c}115 \pm 30 \\
(80-160)\end{array}$ & 5 & $(-10.0,20.0)$ & $=0.5$ \\
\hline - Estimated blood loss $(\mathrm{ml})^{*}$ & $\begin{array}{c}480 \pm 70 \\
(340-750)\end{array}$ & $\begin{array}{l}520 \pm 120 \\
(350-750)\end{array}$ & 40 & $(-2.0,82.0)$ & $=0.06$ \\
\hline - ASA, I/II & $30 / 13$ & $29 / 15$ & & & \\
\hline
\end{tabular}

Abbreviation:- TAP: Transversus abdominis plane, LWI: Local anesthetic wound infiltration, TAH: Total abdominal hysterectomy, BMI: Body mass index, PMB: Perimenopausal bleeding, ASA: American society of anesthesiologists' status. $\Delta$ : Mean difference, $95 \%$ CI: 95\% confidence interval.

- Values were given as mean \pm standard deviation (range)* or number (percentage) $* *$

$-\mathrm{P}<0.05$ : statistically significant.

Table (2):- Pain difference at rest in TAP block versus LWI for post TAH pain trial.

\begin{tabular}{|c|c|c|c|c|c|}
\hline $\begin{array}{c}\text { VAS score } \\
(0=\text { non, } 100=\text { worst })\end{array}$ & $\begin{array}{c}\text { TAP } \\
(n=43)\end{array}$ & $\begin{array}{c}\text { LWI } \\
(n=44)\end{array}$ & $\begin{array}{c}\Delta \\
\text { mean }\end{array}$ & 95\% C.I & $P$ value \\
\hline PACU* & $\begin{array}{l}32 \pm 10 \\
(24-75)\end{array}$ & $\begin{array}{l}38 \pm 12 \\
(28-90)\end{array}$ & 6 & $(1.2,10.2)$ & $=0.01$ \\
\hline 2 hour* & $\begin{array}{c}36 \pm 8 \\
(26-70) \\
\end{array}$ & $\begin{array}{l}44 \pm 10 \\
(28-80)\end{array}$ & 8 & $(4.1,11.8)$ & $=0.0001$ \\
\hline 4 hour* & $\begin{array}{c}36 \pm 16 \\
(28-75) \\
\end{array}$ & $\begin{array}{c}40 \pm 18 \\
(30-90)\end{array}$ & 4 & $(-3.2,11.2)$ & $=0.2$ \\
\hline 8 hour* & $\begin{array}{c}38 \pm 18 \\
(30 \pm 90)\end{array}$ & $\begin{array}{c}48 \pm 70 \\
(30-90)\end{array}$ & 10 & $(1.8,18.1)$ & $=0.01$ \\
\hline 12 hour* & $\begin{array}{l}45 \pm 16 \\
(35-85)\end{array}$ & $\begin{array}{c}50 \pm 18 \\
(35-85)\end{array}$ & 5 & $(-2.0,12.7)$ & $=0.1$ \\
\hline 24 hour* & $\begin{array}{c}48 \pm 18 \\
(30-85)\end{array}$ & $\begin{array}{l}50 \pm 14 \\
(40-90)\end{array}$ & 2 & $(-4,8,8.8)$ & $=0.5$ \\
\hline
\end{tabular}

Abbreviation: TAP: Transversus abdominis plane, LWI: Local anesthetic wound infiltration, TAH: Total abdominal hysterectomy, $\Delta$ : Mean difference, $95 \%$ CI: 95\% confidence interval, VAS: Visual analogue scale score, PACU: Post anesthesia care unit.

- Values were given as mean \pm standard deviation (range)*

- P < 0.05: statistically significant. 
Table (3):- Pain difference on movement (coughing, hip flection, walking) in TAP block versus LWI in post TAH pain trial.

\begin{tabular}{|c|c|c|c|c|c|}
\hline $\begin{array}{c}\text { VAS score } \\
(\mathbf{0 =} \text { non, 100 = worst })\end{array}$ & $\begin{array}{c}\text { TAP } \\
(\mathbf{n = 4 3})\end{array}$ & $\begin{array}{c}\text { LWI } \\
(\mathbf{n = 4 4 )}\end{array}$ & $\begin{array}{c}\boldsymbol{\Delta} \\
\text { mean }\end{array}$ & $\mathbf{9 5 \%}$ C. I & P value \\
\hline 2 hours & $38 \pm 12(31-85)$ & $54 \pm 14(31-90)$ & 16 & $(10.4,21.1)$ & $<0.0001$ \\
\hline 4 hours & $40 \pm 10(33-80)$ & $56 \pm 14(33-92)$ & 16 & $(10.8,21.1)$ & $<0.0001$ \\
\hline 8 hours & $42 \pm 16(32-85)$ & $54 \pm 16(32-88)$ & 12 & $(5.1,18.8)$ & $=0.0007$ \\
\hline 12 hours & $41 \pm 18(29-89)$ & $53 \pm 15(35-85)$ & 12 & $(4.9,14.5)$ & $=0.0011$ \\
\hline 24 hours & $48 \pm 19(28-86)$ & $51 \pm 17(27-87)$ & 3 & $(-4.08,10.6)$ & $=0.4$ \\
\hline
\end{tabular}

Abbreviation: TAP: Transversus abdominis plane, LWI: Local anesthetic wound infiltration, TAH: Total abdominal hysterectomy, $\Delta$ : Mean difference, $95 \%$ CI: $95 \%$ confidence interval, VAS: Visual analogue scale score.

- Values were given as mean \pm standard deviation (range)*

- P $<0.05$ : statistically significant.

Table (4):- Analgesics consumption difference in TAP block versus LWI for post TAH pain trial.

\begin{tabular}{|c|c|c|c|c|c|}
\hline Outcome & $\begin{array}{c}\text { TAP } \\
(n=43)\end{array}$ & $\begin{array}{c}\text { LWI } \\
(n=44)\end{array}$ & $\begin{array}{c}\Delta \\
\text { mean }\end{array}$ & $95 \%$ C. I & $P$ value \\
\hline \multicolumn{6}{|l|}{$\begin{array}{lcc}\text { Post } & \text { TAH } & \text { cumulative } \\
\text { Nalbuphine consumption }(\mathrm{mg})^{*}:\end{array}$} \\
\hline at 2 hours & $\begin{array}{l}18.5 \pm 7.9 \\
(10-30) \\
\end{array}$ & $\begin{array}{l}28.6 \pm 5.2 \\
(15-35) \\
\end{array}$ & 10.1 & $(7.2,12.9)$ & $<0.0001$ \\
\hline at 4 hours & $\begin{array}{l}21.8 \pm 6.2 \\
(10-35) \\
\end{array}$ & $\begin{array}{l}29.5 \pm 7.6 \\
(15-45) \\
\end{array}$ & 7.7 & $(4.7,10.6)$ & $<0.0001$ \\
\hline at 8 hours & $\begin{array}{l}26.5 \pm 8.6 \\
(10-45) \\
\end{array}$ & $\begin{array}{l}35.6 \pm 9.6 \\
(10-55)\end{array}$ & 9.1 & $(5.2,12.9)$ & $<0.0001$ \\
\hline at 24 hours & $\begin{array}{l}31.9 \pm 7.6 \\
(10-55)\end{array}$ & $\begin{array}{c}46.2 \pm 5.8 \\
(15-60)\end{array}$ & 14.3 & $(11.3,17.2)$ & $<0.0001$ \\
\hline at discharge & $\begin{array}{c}34.7 \pm 7.2 \\
(10-80\end{array}$ & $\begin{array}{l}51.6 \pm 6.8 \\
(75-90)\end{array}$ & 15.9 & $(13.9,19.8)$ & $<0.0001$ \\
\hline \multicolumn{6}{|l|}{$\begin{array}{l}\text { Post TAH cumulative parenteral } \\
\text { NSAIDs consumption }(\mathrm{mg})^{*} \text { : }\end{array}$} \\
\hline at 2 hours & $\begin{array}{l}22.5 \pm 5.6 \\
(20-40) \\
\end{array}$ & $\begin{array}{l}29.6 \pm 7.8 \\
(20-40) \\
\end{array}$ & 7.1 & $(4.01,10.1)$ & $<0.0001$ \\
\hline at 4 hours & $\begin{array}{l}25.6 \pm 7.8 \\
(20-40) \\
\end{array}$ & $\begin{array}{l}35.6 \pm 6.2 \\
(20-40) \\
\end{array}$ & 10 & $(7.0,12.9)$ & $<0.0001$ \\
\hline at 8 hours & $\begin{array}{l}28.9 \pm 5.6 \\
(20-40)\end{array}$ & $\begin{array}{l}38.6 \pm 7.7 \\
(20-40)\end{array}$ & 9.7 & $(6.8,12.5)$ & $<0.0001$ \\
\hline at 12 hours & $\begin{array}{l}30.6 \pm 9.5 \\
(20-60) \\
\end{array}$ & $\begin{array}{c}40.6 \pm 8.6(20 \\
-60) \\
\end{array}$ & 10 & $(6.1,13.8)$ & $<0.0001$ \\
\hline at 24 hours & $\begin{array}{l}46.9 \pm 9.6 \\
(20-80)\end{array}$ & $\begin{array}{c}50.6 \pm 8.6 \\
(20-80) \\
\end{array}$ & 3.7 & $(-0.1,7.5)$ & $=0.06$ \\
\hline at discharge & $\begin{array}{l}112.6 \pm 21.8 \\
(60-160)\end{array}$ & $\begin{array}{c}115.8 \pm 22.6 \\
(80-160\end{array}$ & 3.2 & $(-6.3,12.7)$ & $=0.5$ \\
\hline
\end{tabular}

Abbreviation: TAP: Transversus abdominis plane, LWI: Local anesthetic wound infiltration, TAH: Total abdominal hysterectomy, NSAIDs: Non-steroidal anti-inflammatory drug, $\Delta$ : Mean difference, 95\% CI: 95\% confidence interval.

- Values were given as mean \pm standard deviation (range)

- P < 0.05: statistically significant. 
Table (5):- Outcomes difference other than pain and analgesics consumption in TAP block versus LWI for post TAH pain trial.

\begin{tabular}{|c|c|c|c|c|c|}
\hline Outcome & $\begin{array}{c}\text { TAP } \\
(n=43)\end{array}$ & $\begin{array}{c}\text { LWI } \\
(n=44)\end{array}$ & $\begin{array}{c}\Delta \\
\text { mean }\end{array}$ & $95 \%$ C. I & P value \\
\hline - Time to get out of bed $(n)^{*}$ & $\begin{array}{c}6.2 \pm 1.9 \\
(4.5-9.5)\end{array}$ & $\begin{array}{c}7.1 \pm 2.1 \\
(3.5-9.5)\end{array}$ & 0.9 & $(0.04,1.75)$ & $=0.03$ \\
\hline -PACU time $(\mathrm{min})^{*}$ & $\begin{array}{c}55 \pm 27 \\
(30-120)\end{array}$ & $\begin{array}{c}50 \pm 25 \\
(40-100)\end{array}$ & -5 & $(-16.0,6.0)$ & $=0.3$ \\
\hline - Hospital stay (h)* & $\begin{array}{c}70 \pm 15 \\
(42-96)\end{array}$ & $\begin{array}{c}68 \pm 18 \\
(38-96)\end{array}$ & -2 & $(-90,5.0)$ & $=0.5$ \\
\hline - Time to first flatus (h)* & $\begin{array}{c}32 \pm 6 \\
(16-58) \\
\end{array}$ & $\begin{array}{c}38 \pm 8 \\
(12-60)\end{array}$ & 6 & $(2.9,9.0)$ & $=0.0002$ \\
\hline - Nausea** & $10(23.2 \%)$ & $12(27.9 \%)$ & $4.7 \%$ & $(-15.0,24.0)$ & $=0.6$ \\
\hline - Vomiting** & $4(9.3 \%)$ & $5(11.3 \%)$ & $2 \%$ & $(-12.8,16.7)$ & $=0.7$ \\
\hline - Pruritis** & $2(4.6 \%)$ & $3(6.8 \%)$ & & & \\
\hline - Patient satisfaction ${ }^{(1)} *$ & $\begin{array}{c}4.1 \pm 0.5 \\
(2-5)\end{array}$ & $\begin{array}{c}3.9 \pm 0.6 \\
(2-5)\end{array}$ & -0.2 & $(-0.4,0.03)$ & $=0.09$ \\
\hline \multicolumn{6}{|l|}{ - Deepest level of sedation ${ }^{(2)} * *$} \\
\hline 0 & $10(23.2 \%)$ & $12(27.9 \%)$ & $4.7 \%$ & $(-15.0,24.0)$ & $=0.61$ \\
\hline 1 & $20(46.5 \%)$ & $18(41.8 \%)$ & $4.7 \%$ & $(-17.3,26.2)$ & $=0.6$ \\
\hline 2 & $13(30.2 \%)$ & $(32.5 \%)$ & $2.3 \%$ & $(18.4,22.7)$ & $=0.8$ \\
\hline
\end{tabular}

Abbreviation: TAP: Transversus abdominis plane, LWI: Local anesthetic wound infiltration, TAH: Total abdominal hysterectomy, $\Delta$ : Mean difference.

95\% CI: 95\% confidence interval, PACU: Post anesthesia care unit.

- Values were given as mean \pm standard deviation (range)* or number (percentage)**

- P < 0.05: statistically significant.

1. Patient satisfaction assessed at 24 hours postoperative using a 5 -point scale $(1=$ very unsatisfied, $2=$ unsatisfied, 3 = fair, $4=$ satisfied, 5 = very satisfied).

2. Deepest level of sedition assessed using a 4-point scale $(0=$ awake and alert, $1=$ minimally sedated, responds to speech, 2 = moderately sedated, arousable by tactile stimulation, 3 = deeply sedated, arousable only by painful stimulation) was recorded in the 24 hours postoperatively and no women in both group was deeply sedated.

\section{Discussion:-}

The immediate postoperative care main objective is to control postoperative pain, nausea, vomiting and promotes early mobilization to avoid development of venous thromboembolism and pneumonia. The advance in acute postoperative pain treatment including patient controlled opioid analgesia, but at risk of its side effects and complications and non-steroidal anti-inflammatory drugs with their ability to minimize dynamic postoperative pain, but also at risk of its complications ${ }^{(17)}$.

This prospective, double-blinded, randomized controlled trial demonstrated that ultrasound guided bilateral transversus abdominis plane block (TAP) with $40 \mathrm{ml}$ bupivacaine $0.25 \%$ is effective in achieving its prespecified primary outcome as it reduces total cumulative postoperative nalbuphine consumption by $16.9 \mathrm{mg}(32.75 \%)(36.7 \pm$ 7.2 vs $51.6 \pm 6.8$ ). also, TAP block is effective than LWI as regards analgesia after TAH as VAS scale was significantly low both at rest and movement at PACU, 2h, $8 \mathrm{~h}(\mathrm{P}<0.05)$ while on movement as well at $4 \mathrm{~h}, 12 \mathrm{~h}$ postoperatively $(\mathrm{P}<0.05)$ as well as reduction of time to get out of bed $(\mathrm{P}=0.03)$ and shorting time to flatus $(\mathrm{P}=$ $0.0002)$.

After careful reviewing medical databases, we couldn't find a trial comparing ultrasound TAP block with local subcutaneous sub facial infiltration in Post TAH pain. However, trial comparing local wound infiltration with TAP block after other abdominal surgery as cesarean section were few ${ }^{(12,13,14,15)}$. Also, meta-analysis of trial comparing TAP block with wound infiltration after abdominal surgery were few ${ }^{(18,19)}$. 
TAP block after TAH compared with placebo is associated with reduced postoperative morphine consumption, improved pain scores at rest and on movement, reduced sedation, as well as increase the time to the first requirement of supplemental analgesia ${ }^{(7)}$. Moreover, TAP block was effective in association with spinal anesthesia for cesarean section postoperative analgesia only if intrathecal morphine isn't used ${ }^{(20,21,22)}$ wound infiltration, with local anesthetic was compared to placebo in transverse pfannenstiel abdominal incisions in cesarean section and found to be associated with lower morphine consumption ${ }^{(23)}$. Also trails of wound infiltration against placebo after TAH found pain scores and morphine consumption was lower when local anesthetic infiltrated after incision in rectus muscle ${ }^{(24)}$. Moreover, subcutaneous and sub facial local anesthetic infiltration after TAH despite didn't reach significant of lower morphine consumption the time to first analgesic request was significantly prolonged ${ }^{(7)}$.

Yu et al. ${ }^{(18)}$ and $\boldsymbol{G u o}$ et $\boldsymbol{a l} .^{(19)}$ assessed randomized trials evaluating TAP block versus wound infiltration. The first meta-analysis ${ }^{(18)}$ assessed four trials conducted on adults undergoing various lower abdominal surgery and they found a significant reduction with TAP block as regards pain scores at 24 hours despite there is no significant difference in pains score at 2 and 4 hours, morphine consumption at 24 hour and incidence of nausea and vomiting.

The second meta-analysis ${ }^{(19)}$ assessed nine studies conducted on different patient groups, including adults, children, parturients undergoing different abdominal procedures, including both laparoscopic and open surgery as TAH, cesarean section and they found that TAP block associated with significant lower pain scores at 8, 24 hours as well as lower morphine consumption at 24 hours but there were no significant differences in pain scores at 1 hour, time to first rescure analgesic, level of sedation and incidence of nausea and vomiting.

As there was no study directly compared TAP block to LWI after TAH under general anesthesia, we will compare our results with available study comparing TAP block with LWI after cesarean section.

Aydogmus et al. ${ }^{(12)}$ using $40 \mathrm{ml}$ levobupivacaine $0.25 \%$ for TAP block or LWI found that TAP block significantly reduced pain scores at 2, 6, 12, 24 hours as well as prolong the time to first analgesic need but no difference was found regards pain scores in first mobilization and patient satisfaction towards postoperative analgesia.

Telnes et al. ${ }^{(13)}$ using $40 \mathrm{ml}, 0.25 \%$ bupivacaine with $5 \mathrm{mg} / \mathrm{ml}$ of Adrenaline for TAP block or LWI and they found no significant difference as regards cumulative morphine consumption as well as pain scores at 12, 24, 36, 48 hours. Also, there were no difference regards time to first analgesic need and incidence of nausea and vomiting.

In this trial we didn't recorded any systemic toxicity ${ }^{(14,23,24)}$ of local anesthetic in both arms of our trial like reported in prematurely terminated study of Chandon et al. ${ }^{(14)}$ due to occurrence of generalized convulsions in women in TAP group. Also, Weiss et al. ${ }^{(24)}$ reported two patients with convulsion after TAP block in cesarean delivery.

The limitations of this trial may be, that we didn't include a control group, however we relayed on the prior evidence of effectiveness of both techniques in comparison to Placebo ${ }^{(7,11,24)}$. We choice total cumulative Nalbuphine consumption as primary outcome, however it represents an overall assessment of postoperative analgesia as well as it does not depend on the experience or tendency of outcome assessor. Nalbuphine was choiced as it is the most commonly used opioid for patient controlled analgesia in our University Hospital.

The strengths points in this trial including blinding of patients and assessors, so detections bias was avoided. Also, we avoided performance bias by the following, enrollment of women and recording of baseline data by single gynecology specialist not included in further steps of study conduct, general anesthesia administration, recording of intra-operation data, preparation of local anesthetics by an anesthesiology specialist not involved in the study and SHAM TAP block procedures. However, Blinding the gynecologist performing LWI and anesthesiologist performing TAP block was not done as it was considered unethical as this necessitating injection of saline in TAP or surgical wound $^{(32)}$.

Tawfik et al. ${ }^{(15)}$ using $30 \mathrm{ml}$ in LWI and $40 \mathrm{ml}$ in TAP block of $0.25 \%$ bupivacine after spinal anesthesia for cesarean delivery and they found no significant difference in postoperative fentanyl consumption and pain scores. 


\section{Conclusion:-}

This prospective randomized trial has shown that ultrasound guided bilateral transversus abdominis plane block with $40 \mathrm{ml} 0.25 \%$ bupivacaine associated with significant reduction in total cumulative Nalbuphine consumption and a lower pain scores at both rest and on movement up to 12 hours postoperatively after TAH undergoing general anesthesia. So, we recommend whenever possible to do TAP block after TAH but when not possible to do at least LWI as part of multimodal postoperative analgesia.

\section{Acknowledgement:-}

The authors want to thank their colleges, fellows, patients, data collectors whom helping them in completing this study.

\section{References:-}

1. Nieboer TE, Johnson, N, Lethaby A, Tavender E, Curr E, Garry R, et al. Surgical approach to hysterectomy for benign gynaecological disease. Cochrane Database syst Rev 2005; (3) : CD005428.

2. Houmes RJ, Voets MA, Verkaaik A, Erdmann W, Lachmann B. Efficacy and safety of tramadol versus morphine for moderate and severe postoperative pain with special regard to respiratory depression. Anesth Analg 1992; $74: 510-514$.

3. Woodhouse A, Mather $\boldsymbol{L E}$. The effect of duration of dose delivery with patient - controlled analgesia on the incidence of nausea and vomiting after hysterectomy. Br J clin Pharmacol 1998; $45: 57$-62.

4. Wang Y, Sands LP, Vaurio L, Mullen EA, Leung JM. The effect of postoperative pain and its management on postoperative cognitive dysfunction. Am J Geriatr Psychiatry, 2007; $15: 50$ - 9 .

5. Kroon UB, Radstrom M, Hjelthe C, Dahlin C, Kroon L. Fast track hysterectomy : a randomized, controlled study. Eur J Obstet Gynecol Reprod Biol 2010; $151: 203$ - 207.

6. Borendal Wodlin N, Nilsson L, Kjolhede P. The impact of mode of anaesthesia on postoperative recovery from fast - track abdominal hysterectomy : a randomized clinical trial. BJOG 2011; $118: 299-208$.

7. Carney J, McDonnell JG, Ochana A, Bhinder R, Laffey JG. The transversus abdominis plane block provides effective postoperative analgesia in patients undergoing total abdominal hysterectomy. Anesth Analg 2008; 107 : 2056 - 2060.

8. Leung CC, Chan YM, Ngai SW, Ng KFJ, Tsui SL. Effect of pre-incision skin infiltration on post-hysterectomy pain - a double- blind randomized controlled trial. Anaesth Intensive Care 2000; $28: 510-516$.

9. Moeniche S, Mikkelsen s, Wetterslev J an Dahl JB. A qualitative systematic review of incisional local anaesthesia for postoperative pain relief after abdominal operations. Br J Anaesth 1998; $81: 377-383$.

10. Leong WM, Lo WK, Chiu JW. Analgesic efficacy of continuous delivery of bupivacaine by an elastomeric balloon infusor after abdominal hysterectomy: a prospective randomized controlled trial. Aust $\mathrm{N} \mathrm{Z} \mathrm{J} \mathrm{Obstet}$ Gynaecol. 2002; $42: 515-518$.

11. Ige OA, Bolaji BO, Kolawole IK: Opioid-sparing effect of bupivacaine wound infiltration after lower abdominal operations. Journal of the west African college of surgeons, 2011; 1 (3): $\quad 62-82$.

12. Aydogmus M, Sinikoglu S, Naki M, Ocak N, Sanh N, Alagol A. Comparison of analgesic efficiency between wound site infiltration and ultra-sound-guided transversus abdominis plane block after cesarean delivery under spinal anaesthesia. Hippokratia, 2014; $18: 28-31$.

13. Telnes A, Skogvoll E, Lonnee H. Transversu abdominis plane block vs. wound infiltration in Caesarean section : a randomized controlled trial. Acta Anaesthesiol Scand. 2015; 59: 496 - 504.

14. Chandon M, Bonnet A, Gurg Y, et al. Ultrasound - guided transversus abdominis plane block versus continuous wound infusion for post-caesarean analgesia: a randomized trial. PLOS One, 2014; 9 : e103971.

15. Tawfik MM, Mohamed YM, Elbadrawi RE, ABdelkhalek M, Mogahed MM, Ezz HM. Transversus abdominis plane block versus wound infiltration for analgesia after cesarean delivery: A randomized controlled Trial. Regional Anesthesia and Acute Pain Medicine, 2017; 124 (4): 1291 -1297.

16. MedCalc Statistical Software version 17.4 (MedCalc Software bvba, Ostend, Belgium from the world web, https://www.medcalc.org;2017.

17. Kehlet H, Dahl JB. The value of multimodal or balanced analgesia in postoperative pain treatment. Anesth Analg. 1993; $77: 1048-56$.

18. 18- Yu N, Long X, Lujan-Hernandez JR, Succar J, Xin X, Wang X: Transversus abdominis - plane block versus local anesthetic wound infiltration in lower abdominal surgery :a systematic review and meta - analysis of randomized controlled trials. BMC Anesthesiol. 2014; $14: 121$. 
19. 19- Guo Q, Li R, Wang L, Zhang D, Ma Y: Transversus abdominis plane block versus local anaesthetic wound infiltration for post-operative analgesia: a systematic review and meta-analysis. Int J Clin Exp Med. 2015; 8 : $17343-17352$.

20. 20- Abdallah FEW, Halpern SH, Margarido CB: Transversus abdominis plane block for postoperative analgesia after caesarean delivery performed under spinal anaesthesia? A systematic review and meta-analysis. Br J Anaesth. 2012; $109: 679-687$.

21. 21- Mishriky BM, George RB, Habib AS: Transversus abdominis plane block for analgesia after Cesarean delivery :a systematic review and meta-analysis. Can J Anaesth. 2012; $59: 766-778$.

22. 22- Fusco P, Scimia P, Paladini G, Fiorenzi M, Petrucci E, Pozone T, et al. : Transversus abdominis plane block for analgesia after Cesarean delivery. A systematic review. Mineroa Anestesiol. 2015; $81: 195$ - 204.

23. 23- Li X, Zhou M, Shi X, Yang H, Li Y, Li J, et al. Local anaesthetic wound infiltration used for caesarean section pain relief: a meta - analysis. Int J Clin Exp Med. 2015; 8 : 10213 - 10224.

24. 24- Tan CH, Kun KY, Onsiong MK, Chan MK, Chiu WKY, Tai CM. Post-incisional local anaesthetic infiltration of the rectus muscle decreases early pain and morphine consumption after abdominal hysterectomy. Acute Pain 2002; $4: 49-52$.

25. 25- Naidu RK, Richebe P: Probable local anesthetic systemic toxicity in a postpartum patient with acute fatty liver of pregnancy after a transversus abdominis plane block. A A Case Rep. 2013; 1: $72-74$.

26. 26- Weiss E, Jolly C, Dumoulin JL, Meftah RB, Blanie P, Laloe PA, et al.. Convulsions in 2 patients after bilateral ultrasound - guided transversus abdominis plane blocks for cesarean analgesia. Reg Anesth Pain Med. 2014; 39:248 - 251 .

27. Cyna AM, Tan SGM. Invasive placebo revisited. Anaesthesia. 2014; $69: 941-944$. 